This item is the archived peer-reviewed author-version of:

\title{
Network structure of functional hippocampal lateralization in birds
}

\section{Reference:}

Jonckers Elisabeth, Güntürkün Onur, de Groof Geert, Van Der Linden Anne-Marie, Bingman Verner P..- Network structure of functional hippocampal lateralization in birds

Hippocampus - ISSN 1050-9631 - 25:11(2015), p. 1418-1428

Full text (Publishers DOI): http://dx.doi.org/doi:10.1002/hipo.22462

To cite this reference: http://hdl.handle.net/10067/1263140151162165141 
Hippocampus

\section{Network Structure of Functional Hippocampal Lateralization} in Birds

\begin{tabular}{|r|l|}
\hline Journal: & Hippocampus \\
\hline Manuscript ID: & Draft \\
\hline Wiley - Manuscript type: & Research Article \\
\hline Keywords: & functional connectivity, resting state fMRI, hemispheric dominance \\
\hline \multicolumn{2}{c}{} \\
& \multicolumn{2}{c}{$\begin{array}{c}\text { SCHOLARONE } \\
\text { Manuscripts }\end{array}$} \\
&
\end{tabular}




\section{Network Structure of Functional Hippocampal Lateralization in Birds}

\section{Running title: Network Origin of Lateralized Hippocampal Function}

Elisabeth Jonckers ${ }^{1 \#}$, Onur Güntürkün ${ }^{2}$, Geert De Groof ${ }^{1}$, Annemie van der Linden ${ }^{1}$, Verner P.

Bingman $^{3 \#} \quad$ " communicating authors

1 Bio-Imaging Lab, University of Antwerp, 2610 Antwerp, Belgium

2 Department of Biopsychology, Institute of Cognitive Neuroscience, Faculty of Psychology, Ruhr-University

Bochum, 44780 Bochum, Germany;

3 Department of Psychology and J.P. Scott Center for Neuroscience, Mind and Behavior, Bowling Green State University, Bowling Green, 43403 Ohio

19 pages; 5 figures

Correspondence to:

Elisabeth Jonckers

Bio-Imaging Lab

Universiteitsplein 1

2610 Wilrijk

Elisabeth.jonckers@uantwerpen.be,

Keywords: functional connectivity, resting state fMRI, hemispheric dominance

Acknowledgements:

The Research Foundation - Flanders; G042002 and G044311N, GDG is a Postdoctoral Fellow

The Hercules Foundation; AUHA0012

NSF; IOS-0922508

Interuniversity Attraction Poles (IAP); 'PLASTOCINE': P7/17

DFG; SFB 874 


\section{$\underline{\text { Abstract }}$}

Functional hemispheric asymmetry is a common feature of vertebrate brain organization, yet little is known about how hemispheric dominance is implemented at the neural level. One notable example of hemispheric dominance in birds is the leading role of the left hippocampal formation in controlling navigational processes that support homing in pigeons. Relying on resting state fMRI analyses (where FC can be determined by placing a reference 'seed' for connectivity in one hemisphere), we show that following seeding in either an anterior or posterior region of the hippocampal formation of homing pigeons and starlings, the emergent functional connectivity maps are consistently larger following seeding of the left hippocampus. Left seedings are also more likely to result in functional connectivity maps that extend to the contralateral hippocampus and outside the boundaries of the hippocampus. The data support the hypothesis that broader functional connectivity is one neural-organizational property that confers, with respect to navigation, functional dominance to the left hippocampus of birds. 
$\underline{\text { Introduction }}$

Hemispheric asymmetries are a ubiquitous property of vertebrate brains (Ocklenburg et al., 2013, Strockens et al., 2013), but whether these emerge from a common neural-organizational origin remains unknown. Although the majority of studies in mammals have concentrated on cortical asymmetries, left-right hippocampal differences are of growing interest. It has long been known that spatial navigation in humans (Maguire et al., 1997, Burgess et al., 2002), and perhaps more general memory processes in rats (Belcheva et al., 2007, Ivanova et al., 2008, Klur et al., 2009), are more strongly associated with the right hippocampus. In line with the observed functional lateralization, larger right hippocampus volumes have been reported in humans (Pedraza et al., 2004, Woolard and Heckers, 2012). In fact, the volumetric asymmetries are already present in preterm neonates (Thompson et al., 2009) and are modified during puberty (Neufang et al., 2009). Because chimpanzees (Freeman et al., 2004) show a similar adult pattern, a larger right hippocampus might be typical for all Hominidae and possibly other mammalian families.

In a series of seminal papers, it was additionally shown that, in mice, inputs from right CA3 prefer to synapse on large mushroom-shaped spines of ipsilateral CA1 neurons with low densities of GluN2B subunits. By contrast, left CA3-neurons make synapses on left CA1 neuron spines, which are small but rich in GluN2B subunits (Kawakami et al., 2003, Shinohara et al., 2008). Due to this asymmetry, left CA3-CA1 synapses produce more pronounced long-term potentiation (Kohl et al., 2011), which strongly contributes to associative, spatial long-term memory (Shipton et al., 2014). ElGaby et al. (El-Gaby et al., 2014) concluded from these data that the left and right hippocampus might be specialized for different kinds of memory processes, with the left more suited for representing novel spatial configurations, while the right hippocampus, with its "synaptic prewiring", more suited for quickly modifying representations of already existing configurations. 
Although lacking a mammalian neocortex, birds also display functional and anatomical brain asymmetries. The asymmetries include those found in the avian hippocampus, which is homologous as a field to the mammalian hippocampus but with a somewhat different internal organization (Reiner et al., 2004, Abellan et al., 2014, Herold et al., 2014). One prominent example is lateralization of spatial navigation. In homing pigeons, the left hippocampus plays a more important role in navigational processes than the right hippocampus. Left, but not right, hippocampal lesions result in an impairment in navigational map and sun compass-based learning (Gagliardo et al., 2001, Gagliardo et al., 2005), as well as the encoding of goal locations based on environmental geometry (Nardi and Bingman, 2007). In association with this left hippocampal dominance, homing pigeons display righteye superiority (input from the right eye prevailing projects to the left cerebral hemisphere) in various spatial tasks as well as during homing (Ulrich et al., 1999, Prior et al., 2002). The occurrence of so-called path cells, whose response properties seem to represent on-going navigational processes, has only been found in the left hippocampus (Siegel et al., 2006). Finally, the left hippocampus is larger than the right in pigeons with homing experience (Mehlhorn et al., 2010). A left hemispheric bias for navigational processes can also be found in migratory songbirds, whose magnetic compass has been reported to be preferentially controlled by the left hemisphere (Wiltschko et al., 2002, Hein et al., 2011). There is also left hemispheric superiority for presumptively hippocampal-dependent spatial memory in food-caching songbirds (Clayton, 1993). In summary, anatomical, lesion and electrophysiological data indicate that the left hippocampus is dominant with respect to controlling aspects of long-distance navigation and spatial memory in both homing pigeons and songbirds.

An important question in cognitive and computational neuroscience is the extent to which the brains of different species employ similar neural-organizational designs to engineer functional hemispheric asymmetries. Discovering such invariant properties in a comparative framework would point to evolutionary conserved or convergent commonalities in neural computation. 
In the context of the current study, the question is what neural organizational property(ies) may convey dominance to the left hippocampus of birds for spatial cognitive processes? One tempting hypothesis is that the dominance of the left hippocampus may be related to a larger or more strongly connected network architecture. Conveniently, recent developments in the application of resting state $\mathrm{fMRI}$ analyses now allows one to investigate network organization in small animals e.g. (Jonckers et al., 2011, De Groof et al., 2013) Therefore, to test our network hypothesis, we employed resting state $\mathrm{fMRI}$ techniques to determine if there are indeed differences in network organization between left and right hippocampus of homing pigeons and a songbird, the starling.

\section{Materials and methods}

\section{$\underline{\text { Animals }}$}

All procedures were performed in accordance with the European guidelines for the care and use of laboratory animals (86/609/EEC) and were approved by the Committee on Animal Care and Use at the University of Antwerp, Belgium.

Eight pigeons (Columba livia) of the Valentia Figurita breed (four male and four female) were measured. The pigeons were kept on a $12 \mathrm{~h} / 12 \mathrm{~h}$ dark/light cycle, and food and water were provided ad libitum. The pigeons were trained to be scanned awake following the protocol of De Groof et al. (De Groof et al., 2013). In addition, 11 female European starlings (Sturnus vulgaris) were measured. The birds were provided with a natural day/night rhythm (following the timing of ambient sunrise and sunset), ad libitum standard bird feed and drinking water. In contrast to the pigeons, the starlings were scanned while under anesthesia. During handling (immobilization), the starlings were anesthetized with $2 \%$ isoflurane (IsoFlo, Abott, Illinois, USA) administered in a mixture of $30 \%$ O2 and $70 \%$ N2. During resting state acquisition, the level was lowered to $1 \%$. A cloacal thermistor was inserted to monitor body temperature and help maintain it at $41.0+/-0.5^{\circ} \mathrm{C}$ by means of feedbackcontrolled, warm-air circuitry (MR-compatible Small Animal Heating System, SA Instruments, Inc.). 
Breathing rate was monitored using a pressure sensitive sensor under the bird (MR-compatible Small Animal Monitoring and Gating System, SA Instruments, Inc). Following completion of scanning, starlings were placed in a separate cage underneath an infrared light until it fully recovered.

fMRI

Resting state fMRI data were recorded using a seven tesla scanner (Bruker, Ettlingen, Germany). For the pigeon imaging, a custom-made circular RF surface antenna (24 mm) was positioned around the head for acquisition and transmission of radio frequency pulses. For the starling imaging, a linear transmit volume coil and a parallel receiver surface array (Bruker) were used. rsfMRI images were acquired using a RAREst sequence with TE $16 / 15 \mathrm{~ms}$ respectively and TR of $2000 \mathrm{~ms}$. Voxel size was $0.17 \times 0.34 \mathrm{~mm}^{2}$ for pigeon and $0.18 \times 0.36 \mathrm{~mm}^{2}$ for starling. Fourteen axial slices with a slice thickness of $0.7 \mathrm{~mm}$ were recorded. 150 repetitions of each image were acquired with a measuring time of approximately five minutes per sequence.

\section{Pre-Processing}

Pre-processing was carried out using the Statistical Parametric Mapping 8 program (SPM 8; http://www.fil.ion.ucl.ac.uk/spm/software/spm8). Initially, all the images were realigned to the first image as a reference. This was carried out using a least squares approach and a 6 parameter (rigid body) spatial transformation. Subsequently, the images were normalized to enable comparison between different animals.

The realigned $\mathrm{fMRI}$ images for each pigeon were normalized to the pigeon brain MRI atlas developed in our lab (Gunturkun et al., 2012) using SPM8. For the starling data, a least square method (affine registration, followed by estimating nonlinear deformations) was applied. For every bird, all the images were repositioned to match with the images of a chosen reference bird that was measured during this study. 
Then, an in-plane smoothing was carried out using a Gaussian Kernel with a Full Width at Half Maximum (FWHM) of $0.36 \times 0.72 \mathrm{~mm}$ for starling and $0.34 \times 0.68 \mathrm{~mm}$ for pigeon. Next, resting-state, time data were linearly detrended and filtered $(0.01-0.1 \mathrm{~Hz})$ using the Resting-State fMRI Data Analysis Toolkit (REST1.7; http://www.restfmri.net/).

\section{$\underline{\text { Processing and statistics }}$}

A seed based approach was used to estimate Functional Connectivity (FC), meaning that whole brain, voxel based FC derived from a defined region-of-interest, or "seed", was calculated. Using REST, time courses were extracted for four seed regions which were located in the left anterior hippocampal formation, right anterior hippocampal formation, left posterior hippocampal formation and right posterior hippocampal formation. Seed regions were positioned at the same locations in both hemispheres within each species, and we attempted to match as closely as possible the seed locations between species (Figures 1 and 4). FC maps were generated in SPM8 by comparing a seed's time course with time courses of all other voxels within a bird's brain using linear regression with the time course of the seed as covariate. Resulting statistical maps showed significantly connected voxels corrected for multiple comparisons. Both a global signal time course as well as motion parameters, resulting from the realignment, were regressed out during this analysis to improve the specificity of the FC correlations (Kalthoff et al., 2010).

To compare local FC in the hippocampal formation of both hemispheres, cluster sizes derived from the single subject FC maps for both the anterior and posterior seed regions were compared using t-tests. Moreover, mean FC within a cluster (a measure of connectivity strength), based on the outcome of the linear regression for the seed based analyses, was compared between hemispheres. To test the relationship between the cluster size in the left hippocampus and difference in cluster size between the left and right hippocampus in starling (see Results), a linear regression analysis was performed using SPSS. 


\section{$\underline{\text { Results }}$}

Pigeon

FC data was acquired in eight trained, awake pigeons (six data sets for each animal). Displayed in Figure $1 \mathrm{~A}$ are the anterior and posterior seed locations shown in sagittal and horizontal sections. The horizontal sections in Figure 1B, mostly composed of hippocampal formation, reveal examples of the pattern of correlated activity following seeding in the four seed sites from four different pigeons. In the examples, it can be seen that left anterior and posterior hippocampal seeding resulted in a larger field of correlated activity compared to right seeding. This impression is confirmed by statistical analyses (Figures 1C and 1D) showing that cluster sizes were significantly larger in the left hippocampus compared to the right hippocampus for the anterior $(t / 7)=2.922$, $p=0.022)$ seed location, and with a strong trend for the posterior seed location $(t(7)=2.336, p=0.052)$. Moreover, when comparing the strength of the voxel correlations within the clusters of the left hemisphere to those of the right hemisphere, mean local FC was significantly higher in left hippocampus for the anterior seed region $(t(7)=5.58, p=0.001)$ and showed a similarly strong trend for the posterior seed region $(t(7)=2.033, p=0.081$ ) (Figure $1 E)$.

For every pigeon 6 scans were acquired spread over three different scanning days to determine the reliability of the results. Figure 2 shows a representative example of the consistency of the results across scans (for the left seed regions), taken from pigeon 1 of Figure 1. Examination of the example figure reveals that local FC was comparable across scans. It is also noteworthy that some of the scans (nine out of the total 48) resulted in correlated voxels in the contralateral hippocampus. Although contralateral signals were irregular and not amenable to statistical analysis, it was striking that contralateral signals were more frequent and cluster sizes were consistently larger in the right hippocampus following left seeding compared to those observed in the left hippocampus following right seeding (see Figure 2-3). 
As a next step we attempted to provide a hippocampal subdivisional breakdown by analyzing the spatial distribution of the correlated voxels from the rsfMRI analyses. To do so we first created a horizontal map of the pigeon hippocampal formation by projecting the subdivisional map of the hippocampus from Herold et al. (2014) onto the horizontal plane and locating it stereotaxically on a transparent version of the 3D, pigeon brain atlas of Güntürkün et al. (2012) (Figure 3). Then, the horizontal plane was subdivided by a grid composed of squares the size of a single FDR-voxel. Individual voxels were then transposed onto this map. Finally, for each voxel position, the average heat map value for all eight pigeons was calculated.

As can be seen in Figure 3, average FC signals after anterior seeding extended bilaterally within the DL-complex and the two DM subdivisions of the hippocampal formation. Consistent with the results of Figure 1, this signal was generally larger following left hippocampal seeding, and it is noteworthy that with left seeding the signal more broadly extended outside the boundaries of the hippocampal formation into hyperpallium apicale of the avian Wulst. After posterior seeding the FC signal was localized mostly within the Tr-complex and the DM-subdivisions of the hippocampal formation, and again, the extent of the signal was larger with left hippocampal seeding.

Figure 4 offers a higher resolution subdivisional-FC map from one pigeon (shown for the left seeds only). To create the figure, we analyzed frontally aligned sections onto which the hippocampal subdivisional map of Herold et al. (2014) was superimposed. As generally seen in the mean FC maps of Figure 3, the example posterior left seed, located primarily in $\operatorname{Tr}$ and DMd, was functionally connected to $\mathrm{Tr}$ and its neighboring lateral and medial cell layers, $\mathrm{DMd}$ and $\mathrm{DMv}$, in both hemispheres. The anterior seed was located in the DL- and DM-substructures, and was functionally connected to DM, DL as well as the hyperpallium apicale of the Wulst in the ipsilateral and contralateral hemispheres. 


\section{$\underline{\text { Starling }}$}

To further explore hemispheric asymmetry in the extent of hippocampal FC, we carried out a similar analysis in starling. However, in contrast to the pigeons, the starling analysis was done on anesthetized birds (there is no behavioral protocol to work with awake starlings), and because no hippocampal subdivisional map is available for starling, we did not carry out a subdivisional breakdown of our FC maps. For the starling, local FC was calculated for two seed locations (anterior and posterior) in the hippocampus of both hemispheres (four seeds in total). The starling seed regions (Figure 5A) were placed to anatomically match as close as possible the seed regions used for the pigeons.

Although belonging to a different family of birds, the starling data revealed a pattern of hemispheric differences in hippocampal functional connectivity that was strikingly similar to the pigeons (Figure 5B-D). Again, cluster sizes were calculated for both seed locations for all animals and compared between hemispheres. Significantly larger cluster sizes (Figure 5C) were recorded in the left hippocampus (following left seeding) compared to the right hippocampus (following right seeding) for both the posterior $(t(10)=2.504, p=0.031)$ and anterior $(t(10)=2.398, p=0.037)$ seed locations. Inspection of Figure 5D suggests the unexpected finding that larger cluster sizes in the left hippocampus were associated with larger cluster-size differences between the left and right hippocampus. This impression was statistically verified as there was a significant correlation between left hemisphere cluster size and left-right cluster-size difference for both the anterior $(p=0.001)$ and posterior $(\mathrm{p}<0.001)$ seed locations (a similarly significant correlation was not found in pigeon). Consistent with the pigeon analysis, we recorded correlated voxels in the contralateral hippocampus with respect to only left seed regions in some of the measurements (e.g., Figure 5B). 


\section{Discussion}

Using analyses of functional connectivity, we found in homing pigeons and starlings that seeding of both the anterior and posterior left hippocampus, compared to right hippocampus seeding, was associated with a more spread FC, and examined in pigeon only (see below), more strongly correlated functional connectivity network in the ipsilateral hippocampal area. In addition, left-seeding was more likely to result in correlated activity in the contralateral hippocampus and even outside the boundaries of the hippocampal formation. Thus, our data reveal a larger, functionally connected left hippocampal network architecture, which could explain, at least in part, the dominance of the left hippocampus for navigational processes in birds. Beyond this, our results also reveal a common design feature that possibly supports hemispheric dominance in mammals. We will discuss these points by first discussing some methodological issues of our study and then by briefly reviewing hemispheric asymmetries of hippocampal and cortical function in the diverse, lateralized systems of humans and other mammals.

\section{Possible Effects of Anesthesia in Starlings and the Possible Confound of a Larger Left Hippocampus}

The pigeons of the current study were behaviorally trained to remain still, enabling us to carry out the scans in awake birds. The same behavioral training could not be applied to the starlings, which were necessarily scanned while anesthetized. Nonetheless, the same broader functional connectivity in the left hippocampus was observed in both species. It is well known that anesthesia influences both long range FC as well as local functional connectivity, which we recorded in our study (Jonckers et al., 2013, Grandjean et al., 2014). Based on local and long-range FC results in rodents, we obtained resting state data in starling using Isoflurane anesthesia at a very low dose (1\%), which is better at revealing FC compared to other, deeper anesthesia protocols. 
Nevertheless, light anesthesia can result in considerable variation in the extent of FC. This is clearly indicated in the starling data of Figure 5D and could be explained by the likelihood that some starlings were more deeply anesthetized during the scans. Interestingly, a higher cluster size in the starling left hemisphere was correlated with more pronounced lateralization. Because local FC is generally larger in awake animals compared to anesthetized animals, it could be that those animals which were experiencing the lightest anesthesia showed the strongest lateralization in FC.

As noted in the Introduction, a larger left hippocampus has been reported in homing pigeons with navigational experience (Mehlhorn et al., 2010). Although unlikely, this observation opens the possibility that the larger FC in the left hippocampus was a trivial consequence of the left hippocampus being larger. We consider this doubtful because the pigeons used in the current study were not homing pigeons (they were from the Valentia Figurita breed) without any flight experience, which based on the homing pigeon work, would be necessary to promote the development of even possible left-right volume differences. More importantly, not only was the FC in the left hippocampus more spread, but it was also associated with stronger connectivity among the correlated voxels in pigeons (Figure 1E, we did not carry out this analysis in starling because there is no evidence of a leftright volume difference in the hippocampus of starlings). Although in principle a larger hippocampus might lead to a broader functional connectivity network, there is no reason why a larger hippocampus would lead to stronger FC. These considerations support our conclusion of a functionally-related larger and more integrated left hippocampal network. 


\section{$\underline{\text { Hippocampal and Cortical Asymmetries in Humans and Other Mammals }}$}

As outlined in the Introduction, the "spatial" right hippocampus of primates has been shown to have a larger volume compared to the left (Pedraza et al., 2004, Thompson et al., 2009, Woolard and Heckers, 2012). In addition, in humans and perhaps rats, the right hippocampus seems to be more heavily recruited during the execution of various spatial tasks (Maguire et al., 1997, Burgess et al., 2002, Belcheva et al., 2007, Ivanova et al., 2008, Klur et al., 2009).

Detailed analyses of intra-hippocampal synaptic connectivity patterns in mice, however, revealed a more complex picture. These analyses demonstrated that CA3-CA1 connections differed between the left and right hippocampi (Kawakami et al., 2003, Shinohara et al., 2008). The differences were used to explain why both sides could support short-term working memory, but only the left hippocampus was able to support long-term memory acquisition for extra-maze, spatial cues (Shipton et al., 2014). However, the left and right hippocampus might also be specialized to support different aspects of spatial coding. Shinohara et al. (2012) demonstrated that mice from enriched environments display elevated gamma oscillations during theta states in the right hippocampus. Given that hippocampal place cells that encode similar spatial positions also usually discharge during the same gamma cycle (Harris et al., 2003), and the proposition that gamma oscillations could bind cell assemblies with overlapping coding properties across hippocampal space (Siegel et al., 2012), it is then conceivable that gamma oscillatory asymmetries could be related to lateralized differences in the extent of spatial ensemble integration within the mammalian hippocampus.

In humans, a hallmark of lateralized processes is hemispheric differences in the extent of activated cortical networks. For example, hearing or reading activates neuronal assemblies that start in auditory and visual areas, and then spread to the left superior temporal sulcus as well as prefrontal regions (Marinkovic et al., 2003, Hauk et al., 2006). More importantly, language related activations spread across a larger cortical area in the functionally dominant left hemisphere compared to the right (Marinkovic et al., 2003, Marinkovic et al., 2011, Hauk et al., 2012). Larger cortical activation is 
also found in the right hemisphere for processes known to be right hemisphere dominant, e.g. spatial reasoning (Vallesi and Crescentini, 2011) and face recognition (Gao et al., 2013). Such asymmetries in the extent of cortical activation may serve to co-activate a larger number of associated nodes in the dominant hemisphere and thus engage a broader functional network (Pulvermuller, 2013). A related aspect is that functionally dominant cortical areas also have more distributed and bilateral perceptual, spatial and motor representations (Mesulam, 1999, Babiloni et al., 2003, Specht, 2014).

\section{A Common Coding of Forebrain Asymmetries in Birds and Mammals}

What could be the functional relevance of forebrain representational asymmetries? As outlined above, lateralized systems in mammals harbor in the dominant hemisphere a more extensive cortical-representational network of, for example, the relevant perceptual (visual) space that includes input from the left and the right hemifield. In addition, hemispheric-dominant systems are connected to a wider network of downstream and upstream structures, which are then co-activated during task execution (Wang et al., 2014). What has been described for neocortex could apply to the mammalian hippocampus as well (see above).

This general pattern of network connectivity also seems to apply to the avian hippocampus. The left hippocampus in birds is more involved in navigational processes (Gagliardo et al., 2001, Bingman et al., 2006, Mehlhorn et al., 2010) while the right hippocampus may be more involved in general memory processes (Bingman et al., 2006). The more extensive functional connectivity found in the left hippocampus of both homing pigeons and starlings can be interpreted as a reflection of navigational processes requiring a larger integrated network of neurons to code for memory/perceptual space and the computation of navigational paths.

Worth noting is the avian hippocampal asymmetry in FC may be derived from asymmetry in the processing of visual inputs. Pigeons have a more bilateral visual field representation of their tectofugal visual system in the left hemisphere. The tectofugal pathway corresponds to the 
extrageniculostriate system of mammals and is the major visual pathway in pigeons. Due to an asymmetrical crossing of ascending tectofugal fibers, only the left hemisphere receives substantial information both from the left and the right eye (Gunturkun et al., 1998), and consequently a much richer, whole-field visual input. Electrophysiological studies support this finding by showing that single units in the left $n$. rotundus (the thalamic node of the tectofugal pathway) and the left entopallium (the recipient telencephalic area of this system) respond to input from both eyes (Folta et al., 2004, Verhaal et al., 2012). In birds, both tectofugal and thalamofugal visual forebrain areas connect to the hippocampal system via different associative telencephalic structures (Shanahan et al., 2013). As a result, a much richer representation of navigationally (hippocampal) relevant landmark and landscape memories may be stored in the "whole-field" left brain hemisphere (Valencia-Alfonso et al., 2009); memories that can be activated by momentary whole-field (right and left eye) visual-perceptual inputs and acted upon by navigational computations. Therefore, we think it is worth considering that the broader functional connectivity of the left hippocampus in pigeons (and starlings) may be derivative of the informational rich, whole-field visual system processing of the left-brain hemisphere.

To the best of our knowledge, the present study is the first to demonstrate that brain asymmetries in birds, as in mammals, are associated with a larger and stronger functionally connected forebrain system within the hemisphere that is dominant for a certain function. Thus, despite the fact that the mammalian and the avian telencephalon have radically different organizations, some basic aspects of neural lateralization are perhaps surprisingly similar. In principle, the similarity could be the result of common ancestry because left-right hemispheric functional differences are older than the phylogenetic separation of mammals and birds (Ocklenburg and Gunturkun, 2012). 
Although speculative, we consider it equally conceivable that the bird-mammal similarity with respect to $\mathrm{FC}$, as an explanation for functional lateralization as we suggest here, could result from convergent evolution; a similarity that may be an inevitable consequence of the dynamic nature of network organizations in which larger numbers of neurons are recruited when a complex operation, e.g., hippocampal spatial navigation or cortical language, is mostly executed by a functional system within a single hemisphere. That indeed a complete sensorimotor circuit can be under unihemispheric control is shown in meta-control experiments in which task execution is governed by a single hemisphere in humans (Levy and Trevarthen, 1976, Urgesi et al., 2005) and pigeons (Adam and Gunturkun, 2009, Unver and Gunturkun, 2014).

\section{$\underline{\text { References }}$}

Abellan A., Desfilis E., Medina L. 2014. Combinatorial expression of Lef1, Lhx2, Lhx5, Lhx9, Lmo3, Lmo4, and Prox1 helps to identify comparable subdivisions in the developing hippocampal formation of mouse and chicken. Frontiers in neuroanatomy 8:59.

Adam R., Gunturkun O. 2009. When one hemisphere takes control: metacontrol in pigeons (Columba livia). PLoS One 4:e5307.

Babiloni C., Carducci F., Del Gratta C., Demartin M., Romani G.L., Babiloni F., Rossini P.M. 2003. Hemispherical asymmetry in human SMA during voluntary simple unilateral movements. An fMRI study. Cortex 39:293-305.

Belcheva I., Tashev R., Belcheva S. 2007. Hippocampal asymmetry in serotonergic modulation of learning and memory in rats. Laterality 12:475-486.

Bingman V.P., Siegel J.J., Gagliardo A., Erichsen J.T. 2006. Representing the richness of avian spatial cognition: properties of a lateralized homing pigeon hippocampus. Rev Neurosci 17:17-28.

Burgess N., Maguire E.A., O'Keefe J. 2002. The human hippocampus and spatial and episodic memory. Neuron 35:625-641.

Clayton N. 1993. Lateralization and unilateral transfer of spatial memory in marsh-tits. J comp Physiol A 171:807-815.

De Groof G., Jonckers E., Gunturkun O., Denolf P., Van Auderkerke J., Van der Linden A. 2013. Functional MRI and functional connectivity of the visual system of awake pigeons. Behavioural brain research 239:43-50.

El-Gaby M., Shipton O.A., Paulsen O. 2014. Synaptic Plasticity and Memory: New Insights from Hippocampal Left-Right Asymmetries. The Neuroscientist : a review journal bringing neurobiology, neurology and psychiatry.

Folta K., Diekamp B., Gunturkun O. 2004. Asymmetrical modes of visual bottom-up and top-down integration in the thalamic nucleus rotundus of pigeons. The Journal of neuroscience : the official journal of the Society for Neuroscience 24:9475-9485.

Freeman H.D., Cantalupo C., Hopkins W.D. 2004. Asymmetries in the hippocampus and amygdala of chimpanzees (Pan troglodytes). Behavioral neuroscience 118:1460-1465. 
Gagliardo A., loale P., Odetti F., Bingman V.P., Siegel J.J., Vallortigara G. 2001. Hippocampus and homing in pigeons: left and right hemispheric differences in navigational map learning. The European journal of neuroscience 13:1617-1624.

Gagliardo A., Vallortigara G., Nardi D., Bingman V.P. 2005. A lateralized avian hippocampus: preferential role of the left hippocampal formation in homing pigeon sun compass-based spatial learning. The European journal of neuroscience 22:2549-2559.

Gao Z., Goldstein A., Harpaz Y., Hansel M., Zion-Golumbic E., Bentin S. 2013. A magnetoencephalographic study of face processing: M170, gamma-band oscillations and source localization. Hum Brain Mapp 34:1783-1795.

Grandjean J., Schroeter A., Batata I., Rudin M. 2014. Optimization of anesthesia protocol for restingstate $\mathrm{fMRI}$ in mice based on differential effects of anesthetics on functional connectivity patterns. Neurolmage.

Gunturkun O., Hellmann B., Melsbach G., Prior H. 1998. Asymmetries of representation in the visual system of pigeons. Neuroreport 9:4127-4130.

Gunturkun O., Verhoye M., De Groof G., Van Der Linden A. 2012. A 3-dimensional digital atlas of the ascending sensory and the descending motor systems in the pigeon brain. Brain StructFunct.

Harris K.D., Csicsvari J., Hirase H., Dragoi G., Buzsaki G. 2003. Organization of cell assemblies in the hippocampus. Nature 424:552-556.

Hauk O., Coutout C., Holden A., Chen Y. 2012. The time-course of single-word reading: evidence from fast behavioral and brain responses. Neurolmage 60:1462-1477.

Hauk O., Davis M.H., Ford M., Pulvermuller F., Marslen-Wilson W.D. 2006. The time course of visual word recognition as revealed by linear regression analysis of ERP data. Neurolmage 30:13831400.

Hein C.M., Engels S., Kishkinev D., Mouritsen H. 2011. Robins have a magnetic compass in both eyes. Nature 471:E11-12; discussion E12-13.

Herold C., Bingman V.P., Strockens F., Letzner S., Sauvage M., Palomero-Gallagher N., Zilles K., Gunturkun O. 2014. Distribution of neurotransmitter receptors and zinc in the pigeon (Columba livia) hippocampal formation: A basis for further comparison with the mammalian hippocampus. The Journal of comparative neurology 522:2553-2575.

Ivanova M., Ternianov A., Belcheva S., Tashev R., Negrev N., Belcheva I. 2008. Hippocampal asymmetry in exploratory behavior to vasoactive intestinal polypeptide. Peptides 29:940947.

Jonckers E., Delgado Y Palacios R., Shah D., Guglielmetti C., Verhoye M., Van der Linden A. 2013 Different anesthesia regimes modulate the functional connectivity outcome in mice. Magn Reson Med.

Jonckers E., Van Audekerke J., De Visscher G., Van Der Linden A., Verhoye M. 2011. Functional connectivity FMRI of the rodent brain: comparison of functional connectivity networks in rat and mouse. PLoSOne 6:e18876.

Kalthoff D., Seehafer J.U., Po C., Wiedermann D., Hoehn M. 2010. Functional connectivity in the rat at 11.7T: Impact of physiological noise in resting state fMRI. Neuroimage Epub ahead of print.

Kawakami R., Shinohara Y., Kato Y., Sugiyama H., Shigemoto R., Ito I. 2003. Asymmetrical allocation of NMDA receptor epsilon2 subunits in hippocampal circuitry. Science 300:990-994.

Klur S., Muller C., Pereira de Vasconcelos A., Ballard T., Lopez J., Galani R., Certa U., Cassel J.C. 2009. Hippocampal-dependent spatial memory functions might be lateralized in rats: An approach combining gene expression profiling and reversible inactivation. Hippocampus 19:800-816.

Kohl M.M., Shipton O.A., Deacon R.M., Rawlins J.N., Deisseroth K., Paulsen O. 2011. Hemispherespecific optogenetic stimulation reveals left-right asymmetry of hippocampal plasticity. Nature neuroscience 14:1413-1415.

Levy J., Trevarthen C. 1976. Metacontrol of hemispheric function in human split-brain patients. J Exp Psychol Hum Percept Perform 2:299-312. 
Maguire E.A., Frackowiak R.S., Frith C.D. 1997. Recalling routes around london: activation of the right hippocampus in taxi drivers. The Journal of neuroscience : the official journal of the Society for Neuroscience 17:7103-7110.

Marinkovic K., Baldwin S., Courtney M.G., Witzel T., Dale A.M., Halgren E. 2011. Right hemisphere has the last laugh: neural dynamics of joke appreciation. Cogn Affect Behav Neurosci 11:113-130.

Marinkovic K., Dhond R.P., Dale A.M., Glessner M., Carr V., Halgren E. 2003. Spatiotemporal dynamics of modality-specific and supramodal word processing. Neuron 38:487-497.

Mehlhorn J., Haastert B., Rehkamper G. 2010 Asymmetry of different brain structures in homing pigeons with and without navigational experience. The Journal of experimental biology 213:2219-2224.

Mesulam M.M. 1999. Spatial attention and neglect: parietal, frontal and cingulate contributions to the mental representation and attentional targeting of salient extrapersonal events. Philos Trans R Soc Lond B Biol Sci 354:1325-1346.

Nardi D., Bingman V.P. 2007. Asymmetrical participation of the left and right hippocampus for representing environmental geometry in homing pigeons. Behavioural brain research 178:160-171.

Neufang S., Specht K., Hausmann M., Gunturkun O., Herpertz-Dahlmann B., Fink G.R., Konrad K. 2009. Sex differences and the impact of steroid hormones on the developing human brain. Cerebral cortex 19:464-473.

Ocklenburg S., Gunturkun O. 2012. Hemispheric asymmetries: the comparative view. Front Psychol 3:5.

Ocklenburg S., Strockens F., Gunturkun O. 2013. Lateralisation of conspecific vocalisation in nonhuman vertebrates. Laterality 18:1-31.

Pedraza O., Bowers D., Gilmore R. 2004. Asymmetry of the hippocampus and amygdala in MRI volumetric measurements of normal adults. Journal of the International Neuropsychological Society : JINS 10:664-678.

Prior H., Lingenauber F., Nitschke J., Gunturkun O. 2002. Orientation and lateralized cue use in pigeons navigating a large indoor environment. The Journal of experimental biology 205:1795-1805.

Pulvermuller F. 2013. Semantic embodiment, disembodiment or misembodiment? In search of meaning in modules and neuron circuits. Brain Lang 127:86-103.

Reiner A., Perkel D.J., Bruce L.L., Butler A.B., Csillag A., Kuenzel W., Medina L., Paxinos G., Shimizu T., Striedter G., Wild M., Ball G.F., Durand S., Gunturkun O., Lee D.W., Mello C.V., Powers A., White S.A., Hough G., Kubikova L., Smulders T.V., Wada K., Dugas-Ford J., Husband S., Yamamoto K., Yu J., Siang C., Jarvis E.D., Avian Brain Nomenclature F. 2004. Revised nomenclature for avian telencephalon and some related brainstem nuclei. The Journal of comparative neurology 473:377-414.

Shanahan M., Bingman V.P., Shimizu T., Wild M., Gunturkun O. 2013. Large-scale network organization in the avian forebrain: a connectivity matrix and theoretical analysis. Front Comput Neurosci 7:89.

Shinohara Y., Hirase H., Watanabe M., Itakura M., Takahashi M., Shigemoto R. 2008. Left-right asymmetry of the hippocampal synapses with differential subunit allocation of glutamate receptors. Proceedings of the National Academy of Sciences of the United States of America 105:19498-19503.

Shipton O.A., El-Gaby M., Apergis-Schoute J., Deisseroth K., Bannerman D.M., Paulsen O., Kohl M.M. 2014 Left-right dissociation of hippocampal memory processes in mice. Proceedings of the National Academy of Sciences of the United States of America 111:15238-15243.

Siegel J.J., Nitz D., Bingman V.P. 2006. Lateralized functional components of spatial cognition in the avian hippocampal formation: evidence from single-unit recordings in freely moving homing pigeons. Hippocampus 16:125-140.

Siegel M., Donner T.H., Engel A.K. 2012. Spectral fingerprints of large-scale neuronal interactions. Nature reviews Neuroscience 13:121-134. 
Specht K. 2014. Neuronal basis of speech comprehension. Hear Res 307:121-135.

Strockens F., Gunturkun O., Ocklenburg S. 2013. Limb preferences in non-human vertebrates. Laterality 18:536-575.

Thompson D.K., Wood S.J., Doyle L.W., Warfield S.K., Egan G.F., Inder T.E. 2009. MR-determined hippocampal asymmetry in full-term and preterm neonates. Hippocampus 19:118-123.

Ulrich C., Prior H., Duka T., Leshchins'ka I., Valenti P., Gunturkun O., Lipp H.P. 1999. Left-hemispheric superiority for visuospatial orientation in homing pigeons. Behavioural brain research 104:169-178.

Unver E., Gunturkun O. 2014. Evidence for interhemispheric conflict during meta-control in pigeons. Behavioural brain research 270:146-150.

Urgesi C., Bricolo E., Aglioti S.M. 2005. Hemispheric metacontrol and cerebral dominance in healthy individuals investigated by means of chimeric faces. Brain Res Cogn Brain Res 24:513-525.

Valencia-Alfonso C.E., Verhaal J., Gunturkun O. 2009. Ascending and descending mechanisms of visual lateralization in pigeons. Philos Trans R Soc Lond B Biol Sci 364:955-963.

Vallesi A., Crescentini C. 2011. Right fronto-parietal involvement in monitoring spatial trajectories. Neurolmage 57:558-564.

Verhaal J., Kirsch J.A., Vlachos I., Manns M., Gunturkun O. 2012. Lateralized reward-related visual discrimination in the avian entopallium. The European journal of neuroscience 35:1337-1343.

Wang D., Buckner R.L., Liu H. 2014. Functional specialization in the human brain estimated by intrinsic hemispheric interaction. The Journal of neuroscience : the official journal of the Society for Neuroscience 34:12341-12352.

Wiltschko W., Traudt J., Gunturkun O., Prior H., Wiltschko R. 2002. Lateralization of magnetic compass orientation in a migratory bird. Nature 419:467-470.

Woolard A.A., Heckers S. 2012. Anatomical and functional correlates of human hippocampal volume asymmetry. Psychiatry research 201:48-53.

\section{Figure Legends}

Figure 1 Local Functional Connectivity (FC) in left and right hippocampus of awake pigeons: Panel A shows the location of the posterior (red) and anterior (blue) seed locations in the sagittal and horizontal plane overlaid on the pigeon MRI atlas

(35). Panel B shows representative examples of local FC from different pigeons in the four different seed locations shown in A. The heat bar depicts FC strength (t-values); top: left and right anterior seed; bottom: left and right posterior seed. The Cpanel shows mean cluster sizes (+/-SEM) across all animals (and scans) for each seed region. Panel D and E display single subject results (mean of 6 scans, each line represents the results from one pigeon) for all seed regions for cluster size and mean connectivity, respectively. 
Figure 2 Reproducibility of Local Functional Connectivity (FC) in the left hippocampus of awake pigeons: The figure shows a representative example (pigeon 1 of Figure 1) of local FC in the left seed locations. The heat bar depicts the number of acquisitions for which each voxel is part of the FC map.

Figure 3: Subdivisional breakdown of FC maps overlaid on an horizontal representation of the pigeon's hippocampal formation subdivisional organization as described by Herold et al. (Herold et al., 2014) and derived from the 3D pigeon brain atlas of Güntürkün et al. (Gunturkun et al., 2012). Abbreviations: DMd: dorsal part of dorsomedial region of the hippocampal formation; DMv: ventral part of dorsomedial region of the hippocampal formation; Tr complex: triangular region of the ventromedial region of the hippocampal formation together with its associated ventrolateral and ventromedial cell layers; DL complex: the dorsolateral region of the hippocampal formation that includes the dorsal and ventral part of dorsolateral region. Panels a-d show the mean extent of local FC, averaged across all pigeons, for seed locations in left anterior (a), right anterior (b), left posterior (c) and right posterior (d) hippocampal formation. Heat map is depicting FC strengths (t-values).

Figure 4: Sections through the pigeon forebrain in frontal plane showing the extent of functional connectivity with seed locations in left posterior and anterior hippocampal formation of one representative pigeon. Heat map depicts FC strengths (t-values). abbreviations: DMd: dorsal part of dorsomedial region of the hippocampal formation; DMv: ventral part of dorsomedial region of the hippocampal formation; Tr: triangular region of the ventromedial region of the hippocampal formation together with its associated ventrolateral (VI) and ventromedial (Vm) cell layers; DL complex now broken down into its the dorsal (DLd) and ventral (DLv) subdivisions; HA, Hyperpallium apicale; HI, Hyperpallium intercalatum; IHA, N. interstitialis hyperpallii apicalis.

Figure 5: Local FC in left and right hippocampus of the starling: The A-panel shows the location of the posterior (red) and anterior (blue) seed locations overlaid on MRI sagittal and horizontal images of the starling brain. Panel B shows representative examples of local FC in the four different seed locations in three different pigeons (the two right seed examples are from the same pigeon). The heat bar depicts FC strength (t-values). The C-panel shows mean cluster sizes (+/SEM) for the different seed regions $\left(^{*}=p<0.05\right)$. Panel $D$ shows single subject results (each line represents the results from one starling) for the different seed regions. 
2

3

4

5

7

8

10

11

12

13

14

15

16

17

18

19

20

21

22

23

24

25

26

27

29

30

31

32

33

34

35

36

37

38

39

40

41

42

43

44

45

46

47

48

49

50

51

52

53

54

55

56

57

58

59

60

John Wiley \& Sons 


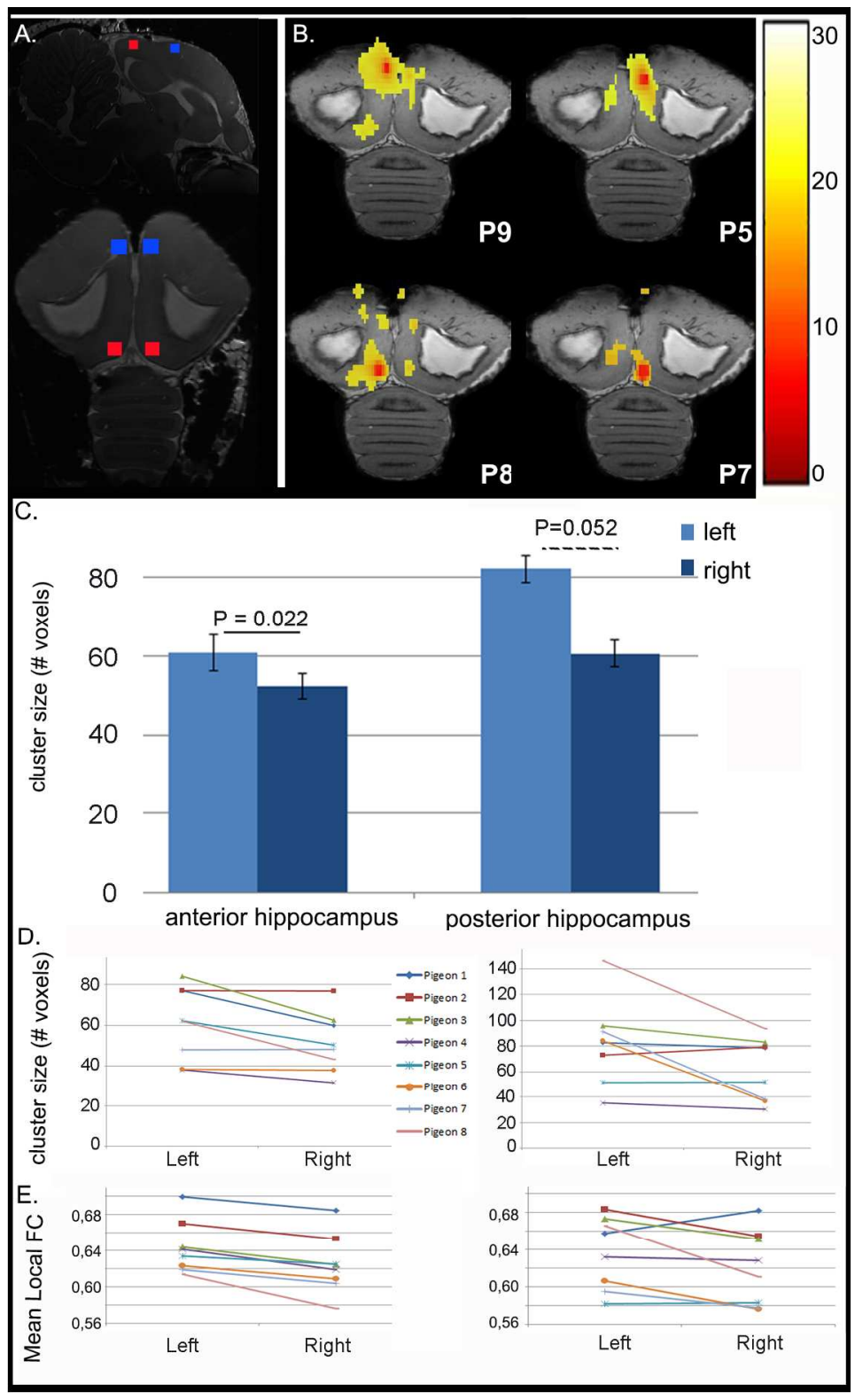

$134 \times 221 \mathrm{~mm}(300 \times 300 \mathrm{DPI})$

John Wiley \& Sons 


\section{Page 23 of 26} Hippocampus

1 2 3

4

5

6
7

8

9

10

11

12

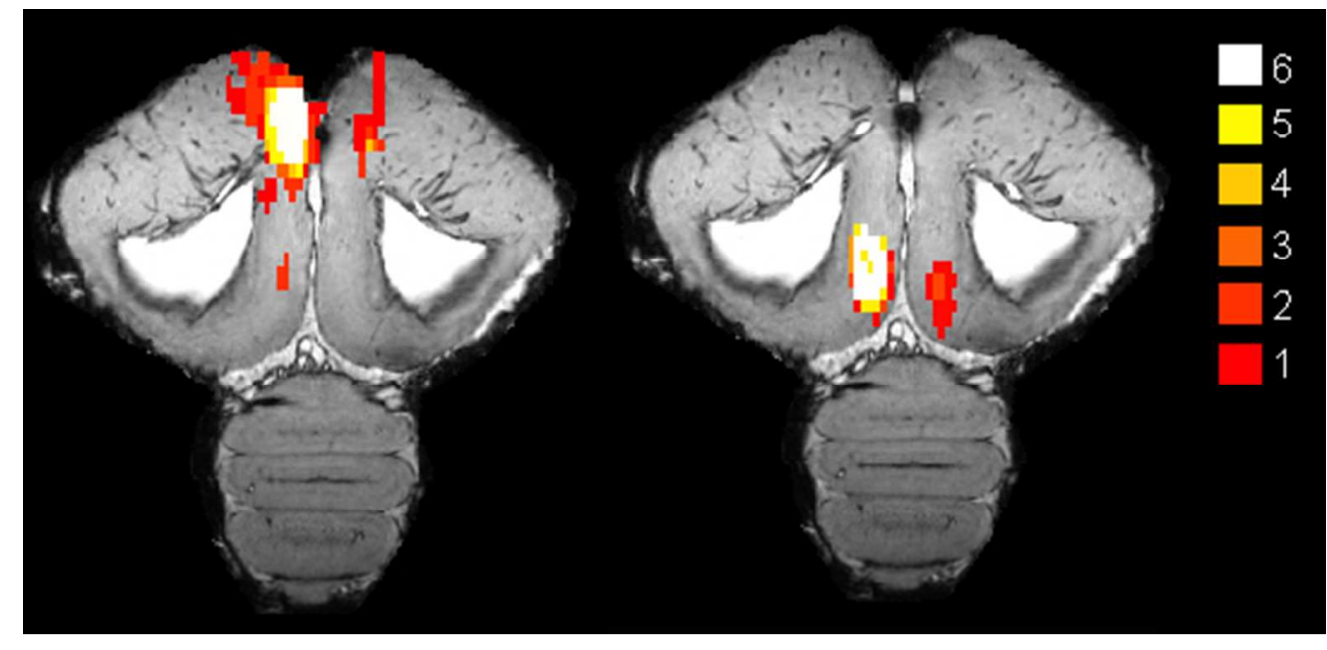

$45 \times 22 \mathrm{~mm}(300 \times 300$ DPI $)$ 


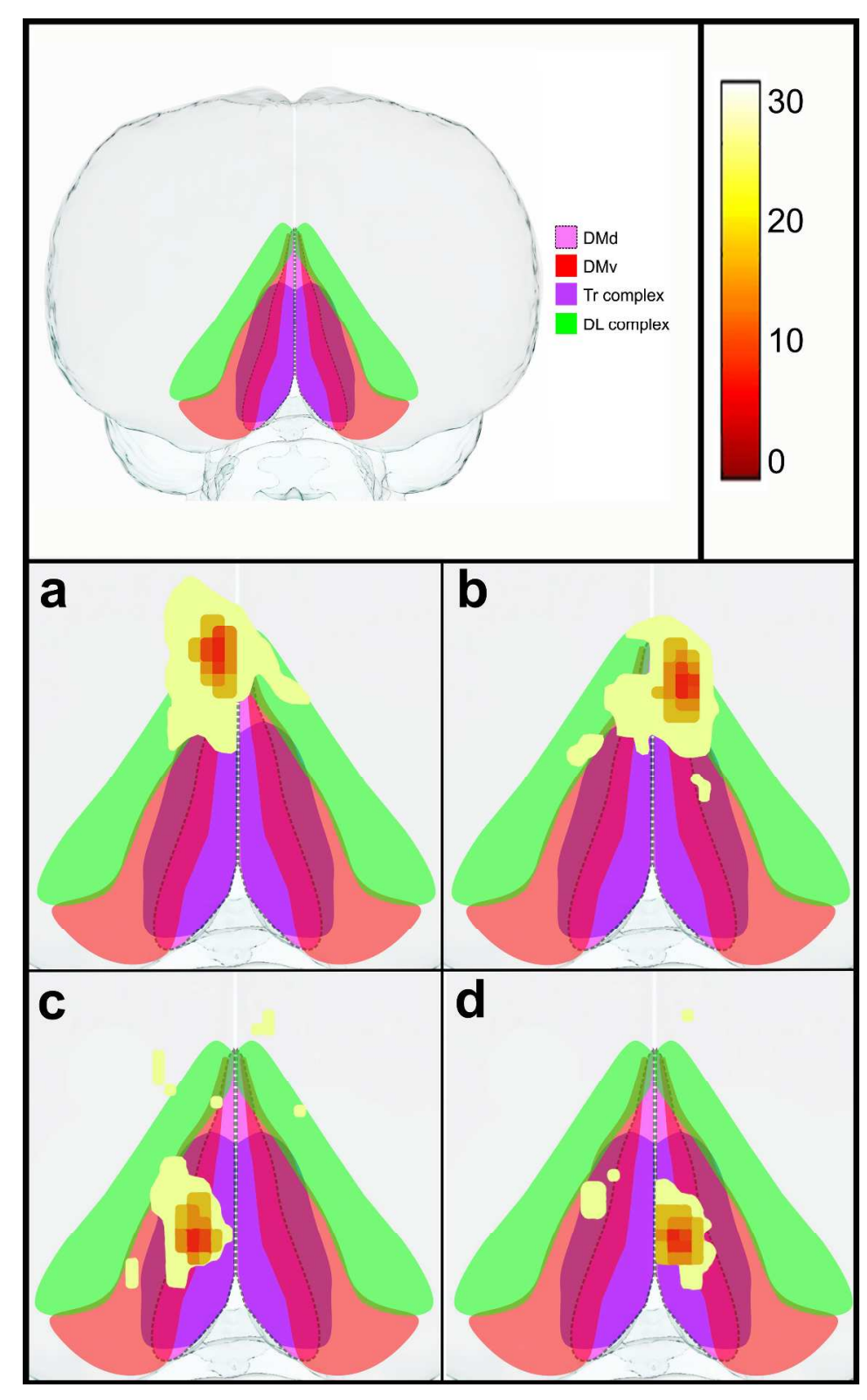

$221 \times 361 \mathrm{~mm}(300 \times 300$ DPI $)$ 

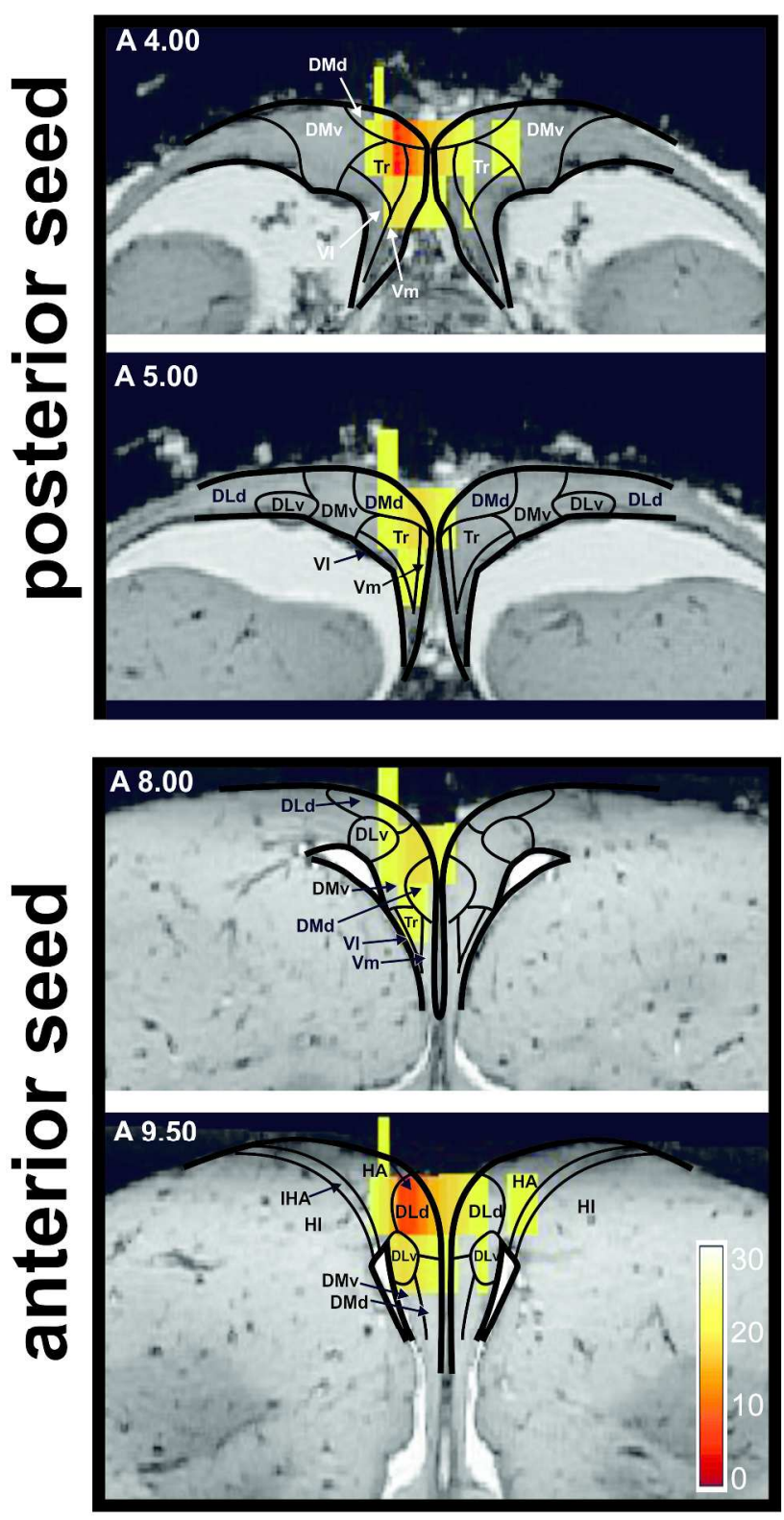

$173 \times 330 \mathrm{~mm}(300 \times 300$ DPI $)$

John Wiley \& Sons 


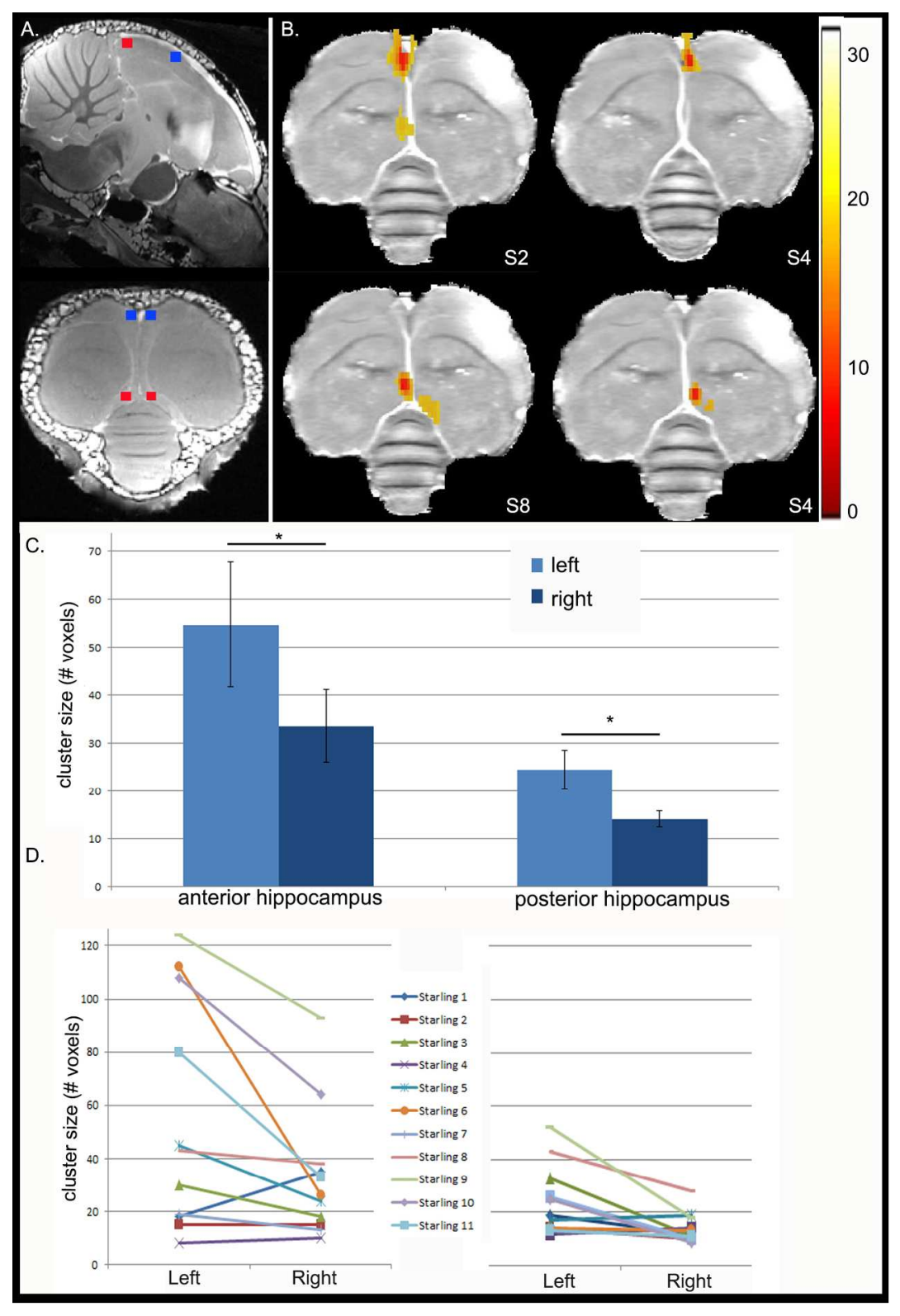

$170 \times 250 \mathrm{~mm}(300 \times 300 \mathrm{DPI})$

John Wiley \& Sons 